\title{
Player A
}
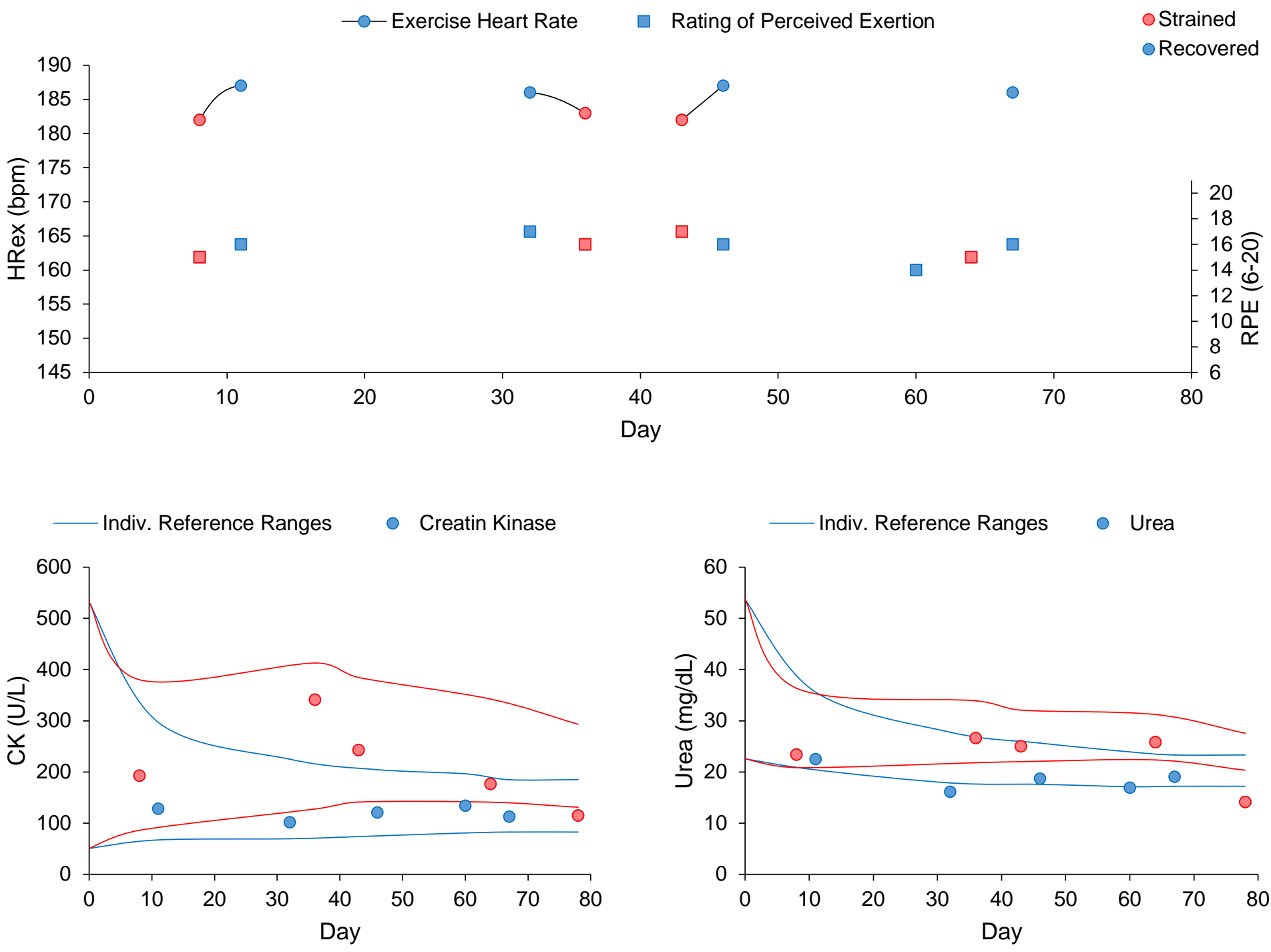

-.-- -.. Physical Perfom. Capability —- Overall Recovery
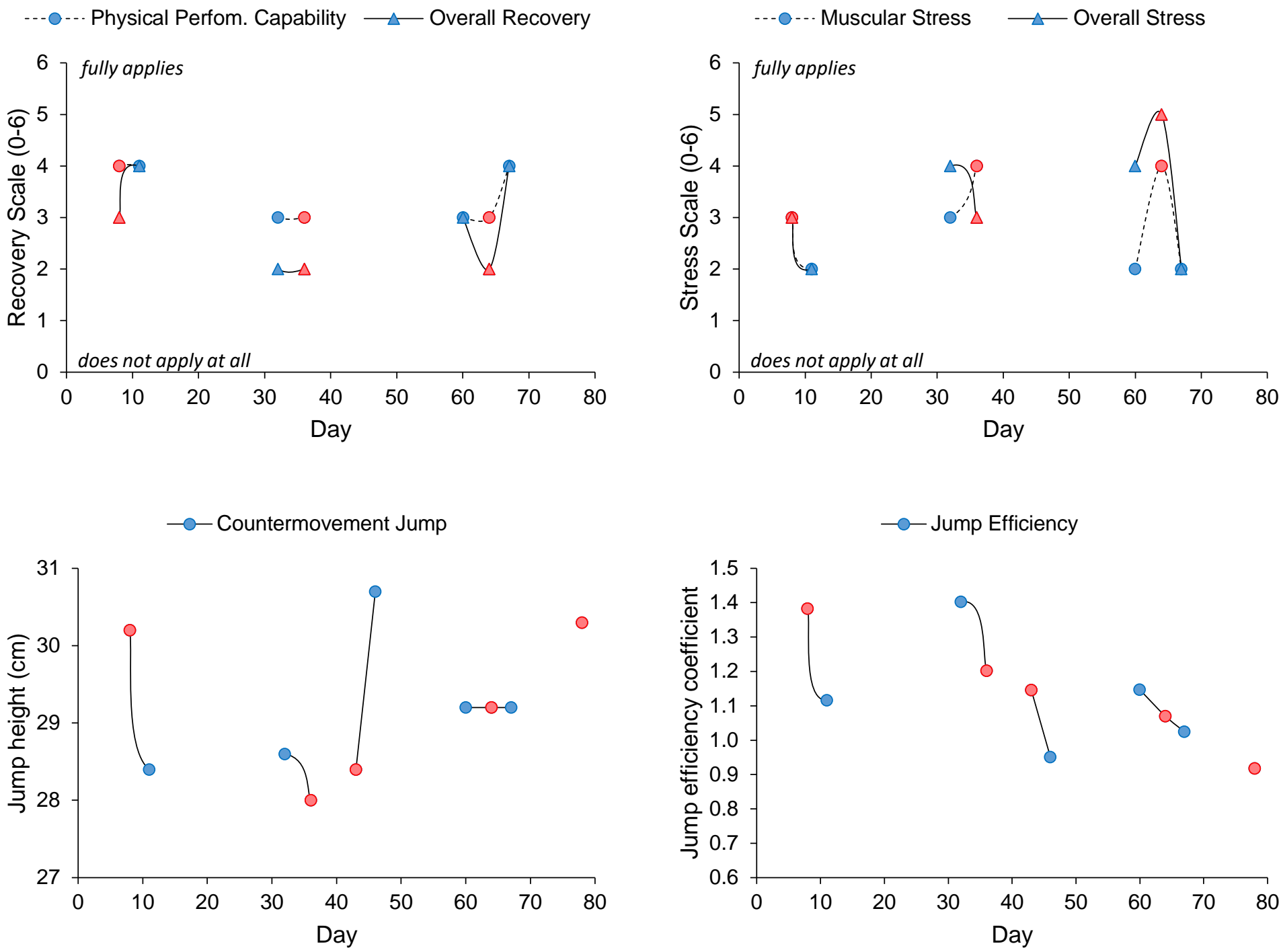

Individual data during the observational period. Blue dots: 'recovered' state (Mondays), red dots: 'strained' state (Fridays). Lines between data points are interrupted if measurements are not available or missing. HRex: exercise heart rate, RPE: rating of perceived exertion, CK: creatine kinase. Plots for CK and Urea also display individualized reference ranges for 'strained' (red lines) and 'recovered' data (blue lines) (95\% confidence intervals), see Hecksteden et al. (2017) for reference and spreadsheets: https://doi.org/10.1123/ijspp.2016-0120 


\section{Player B}
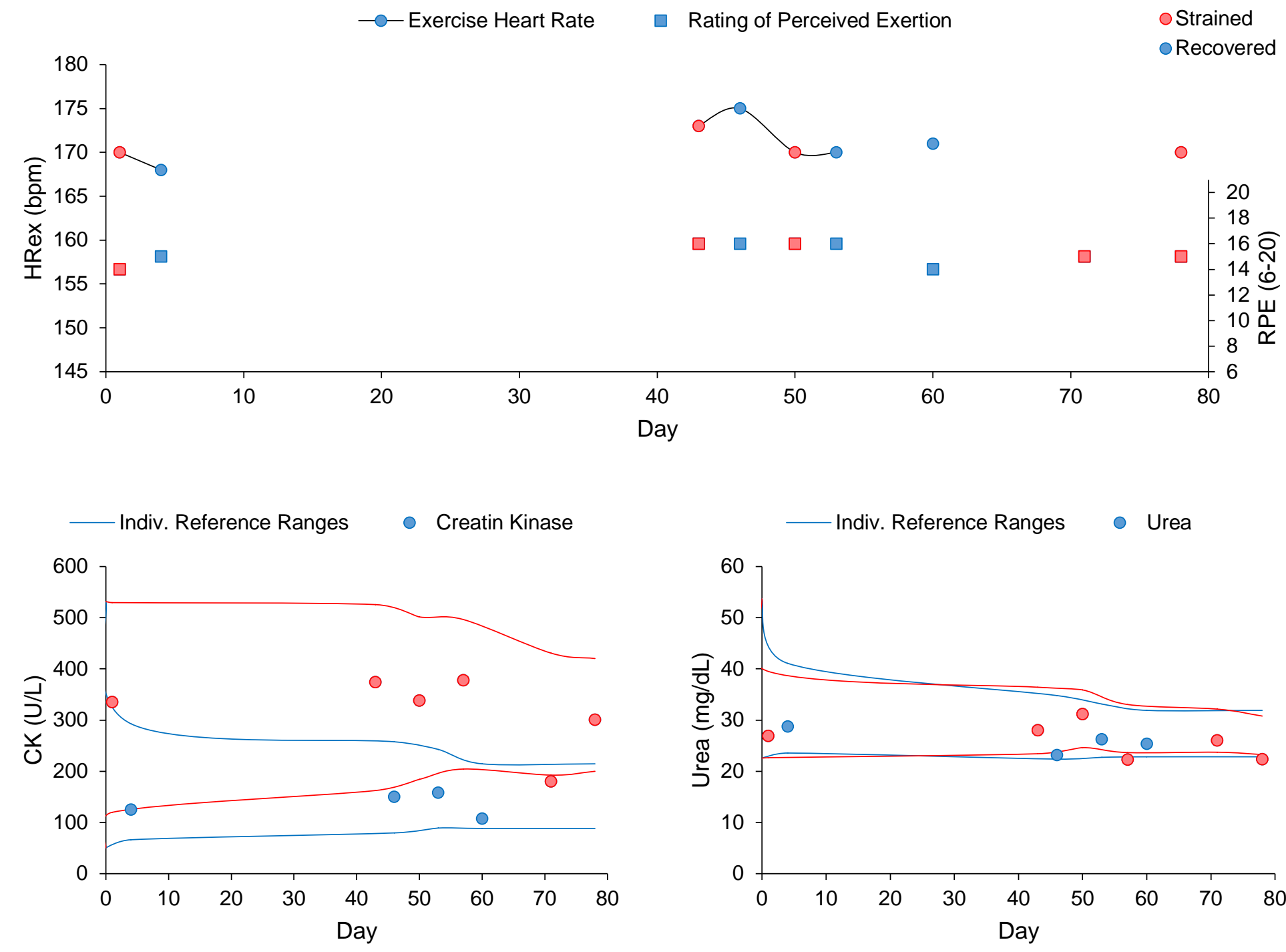

-.--..- Physical Perfom. Capability —-Overall Recovery
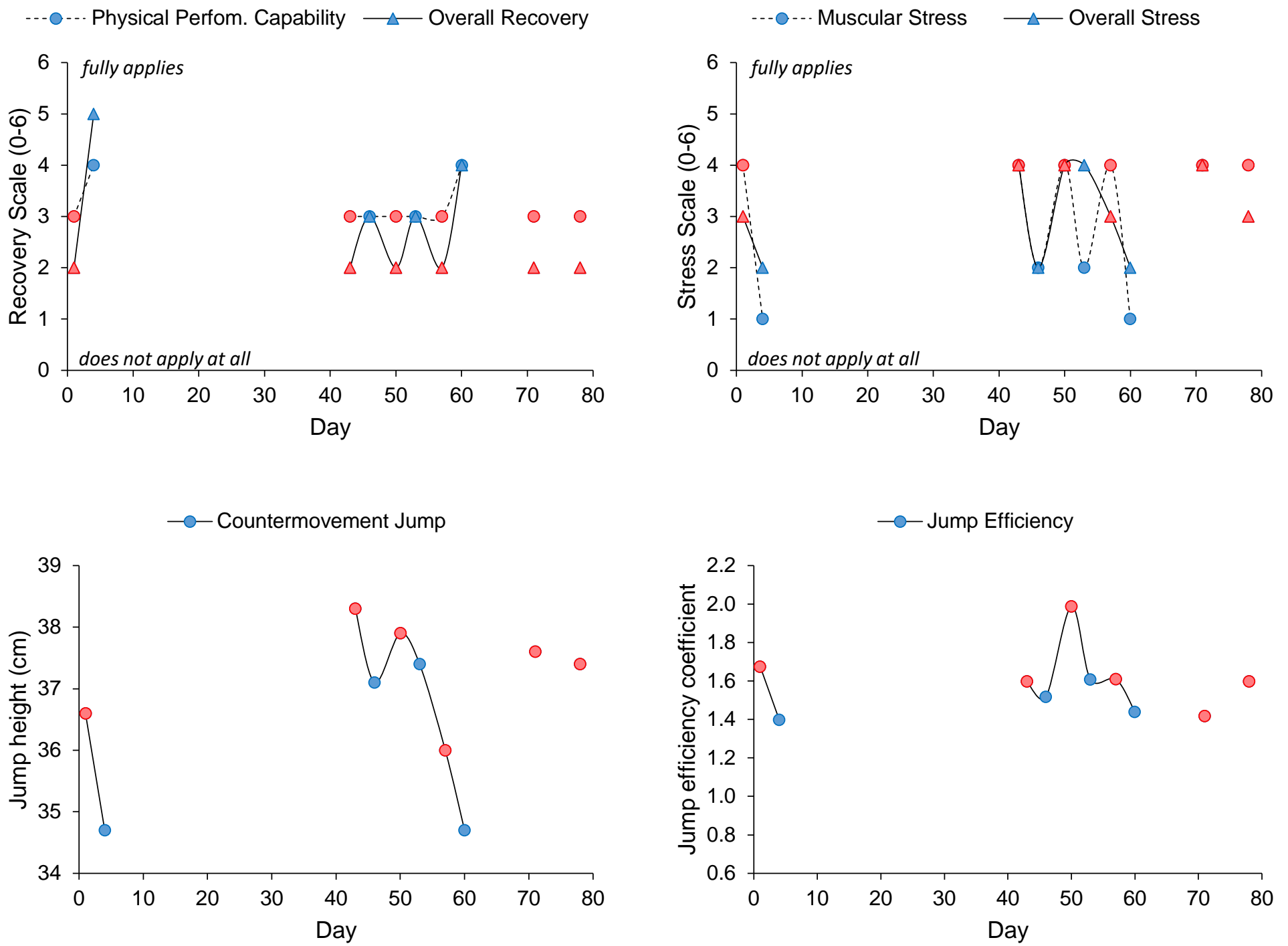

Individual data during the observational period. Blue dots: 'recovered' state (Mondays), red dots: 'strained' state (Fridays). Lines between data points are interrupted if measurements are not available or missing. HRex: exercise heart rate, RPE: rating of perceived exertion, CK: creatine kinase. Plots for CK and Urea also display individualized reference ranges for 'strained' (red lines) and 'recovered' data (blue lines) (95\% confidence intervals), see Hecksteden et al. (2017) for reference and spreadsheets: https://doi.org/10.1123/ijspp.2016-0120 


\section{Player C}
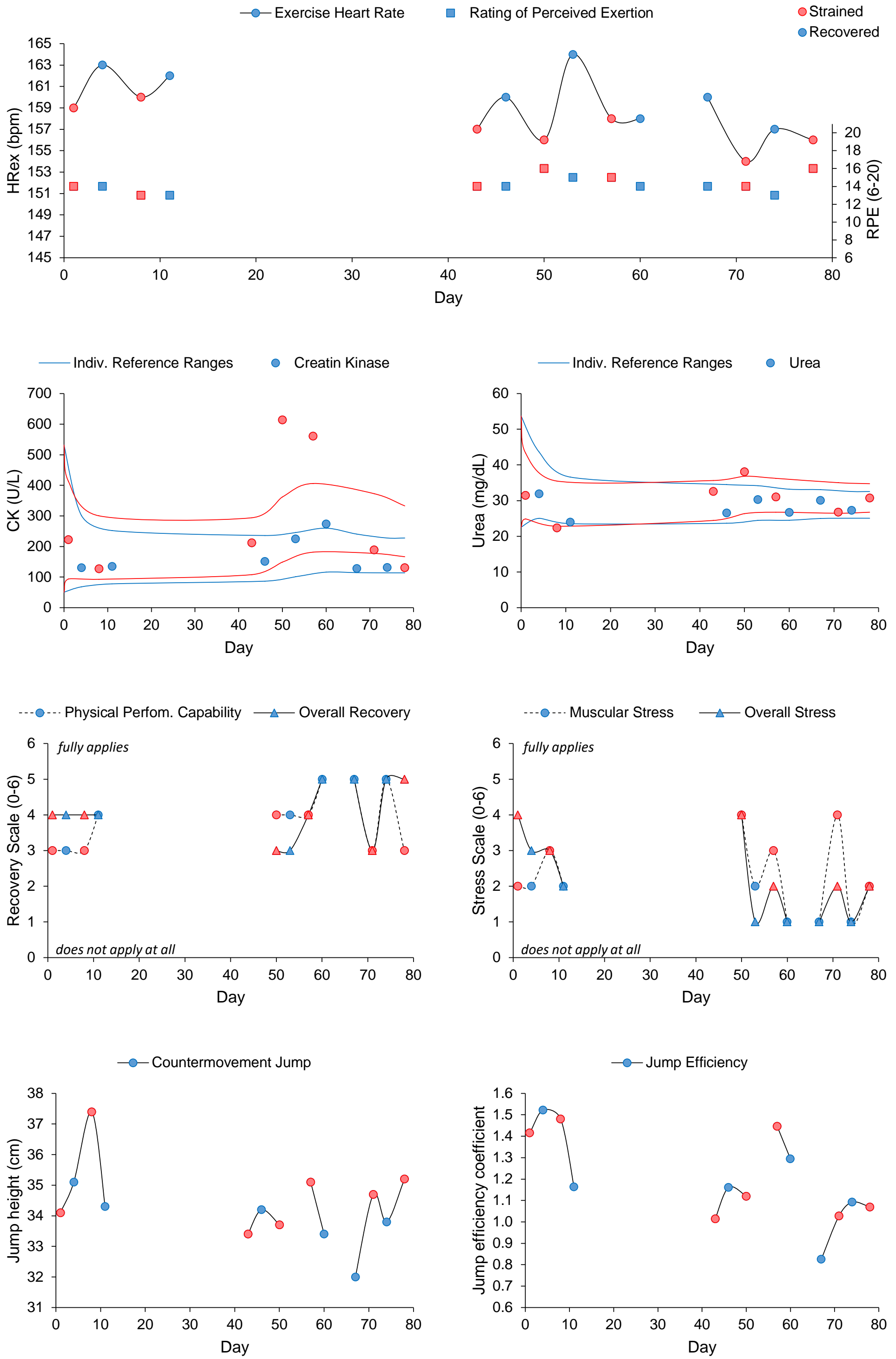

Individual data during the observational period. Blue dots: 'recovered' state (Mondays), red dots: 'strained' state (Fridays). Lines between data points are interrupted if measurements are not available or missing. HRex: exercise heart rate, RPE: rating of perceived exertion, CK: creatine kinase. Plots for CK and Urea also display individualized reference ranges for 'strained' (red lines) and 'recovered' data (blue lines) (95\% confidence intervals), see Hecksteden et al. (2017) for reference and spreadsheets: https://doi.org/10.1123/ijspp.2016-0120 


\section{Player D}
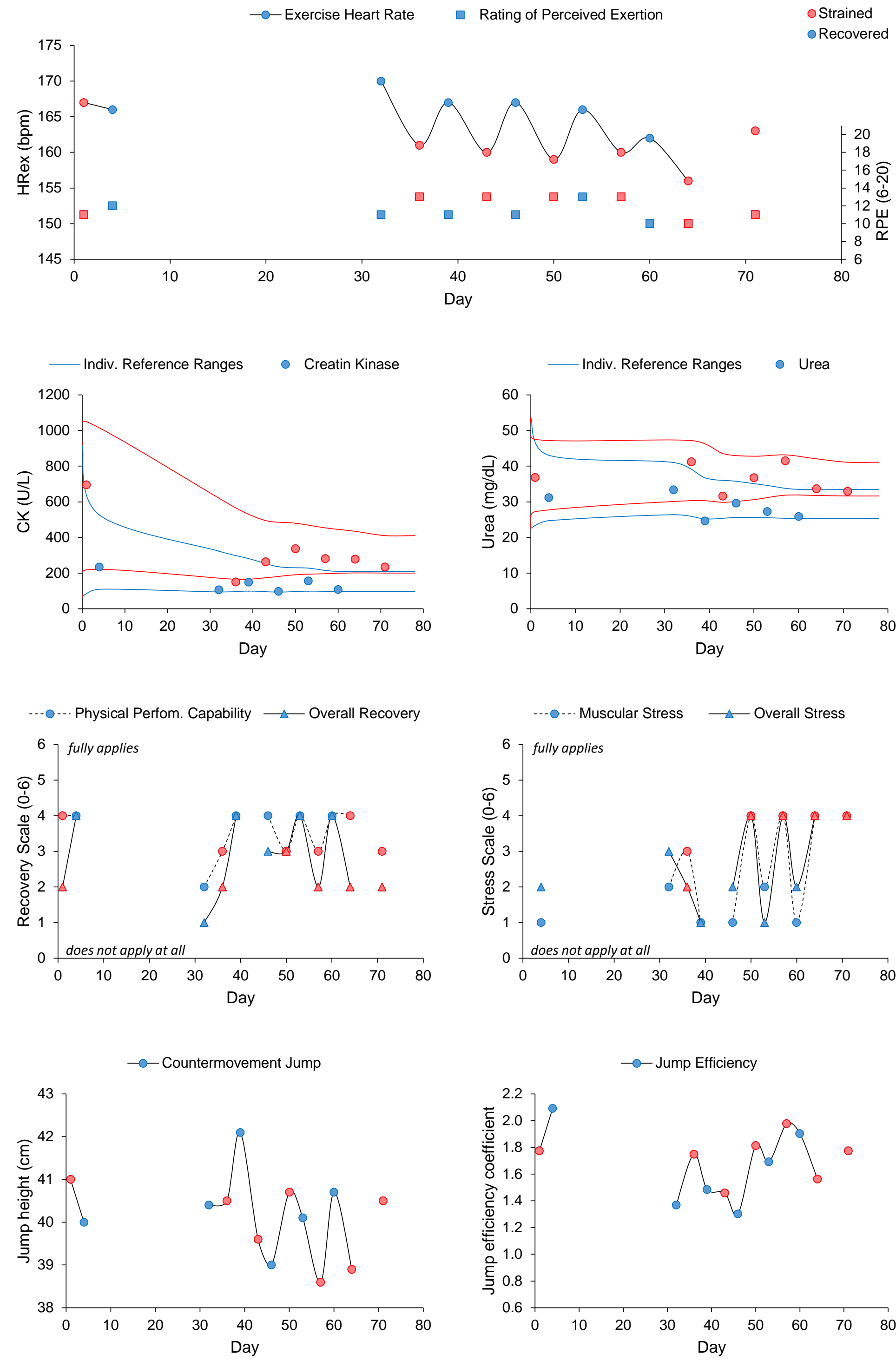

Individual data during the observational period. Blue dots: 'recovered' state (Mondays), red dots: 'strained' state (Fridays). Lines between data points are interrupted if measurements are not available or missing. HRex: exercise heart rate, RPE: rating of perceived exertion, CK: creatine kinase. Plots for CK and Urea also display individualized reference ranges for 'strained' (red lines) and 'recovered' data (blue lines) (95\% confidence intervals), see Hecksteden et al. (2017) for reference and spreadsheets: https://doi.org/10.1123/ijspp.2016-0120 


\section{Player E}
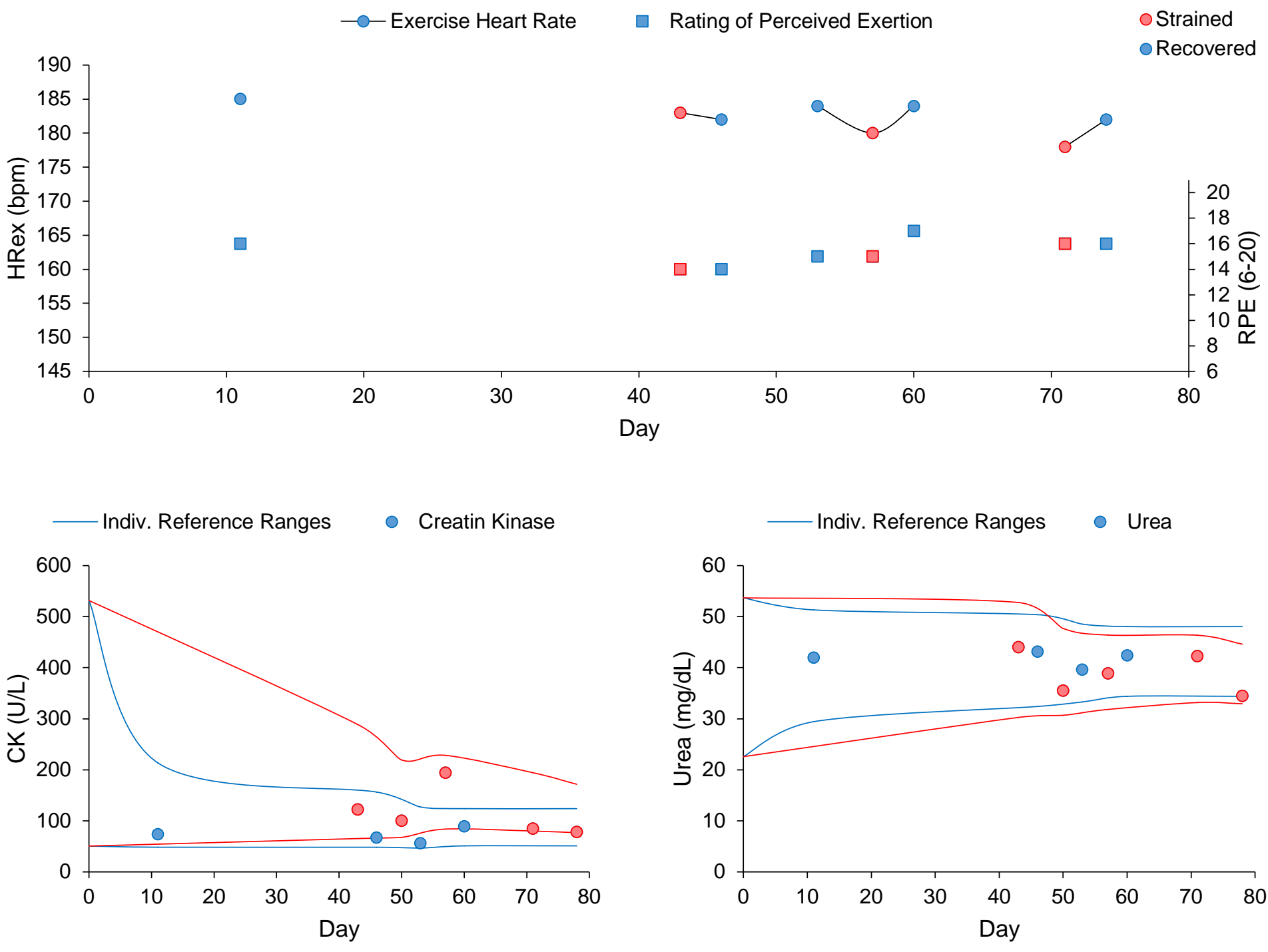

---o --. Physical Perfom. Capability $\longleftarrow$ Overall Recovery
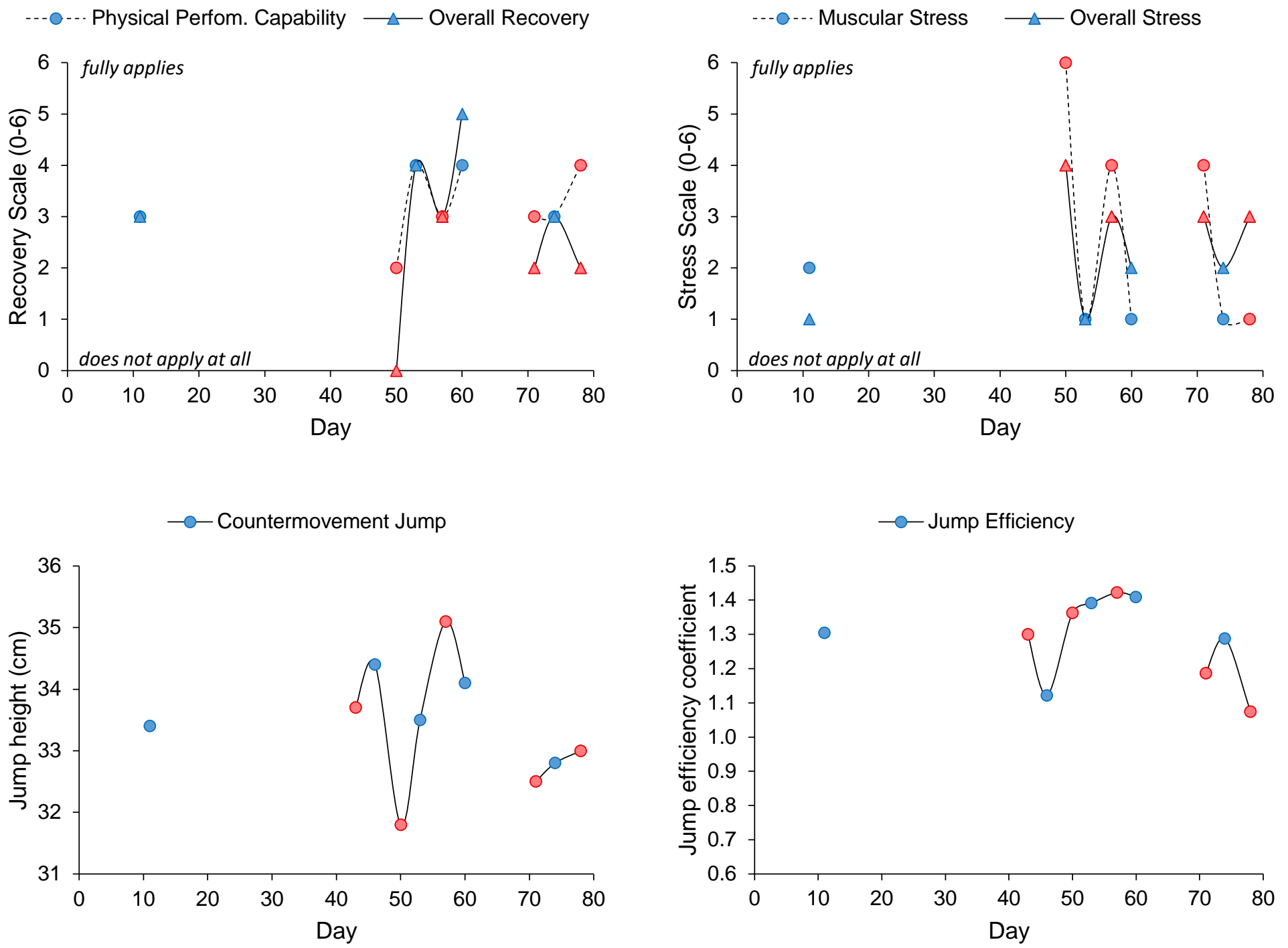

Individual data during the observational period. Blue dots: 'recovered' state (Mondays), red dots: 'strained' state (Fridays). Lines between data points are interrupted if measurements are not available or missing. HRex: exercise heart rate, RPE: rating of perceived exertion, CK: creatine kinase. Plots for CK and Urea also display individualized reference ranges for 'strained' (red lines) and 'recovered' data (blue lines) (95\% confidence intervals), see Hecksteden et al. (2017) for reference and spreadsheets: https://doi.org/10.1123/ijspp.2016-0120 


\section{Player F}
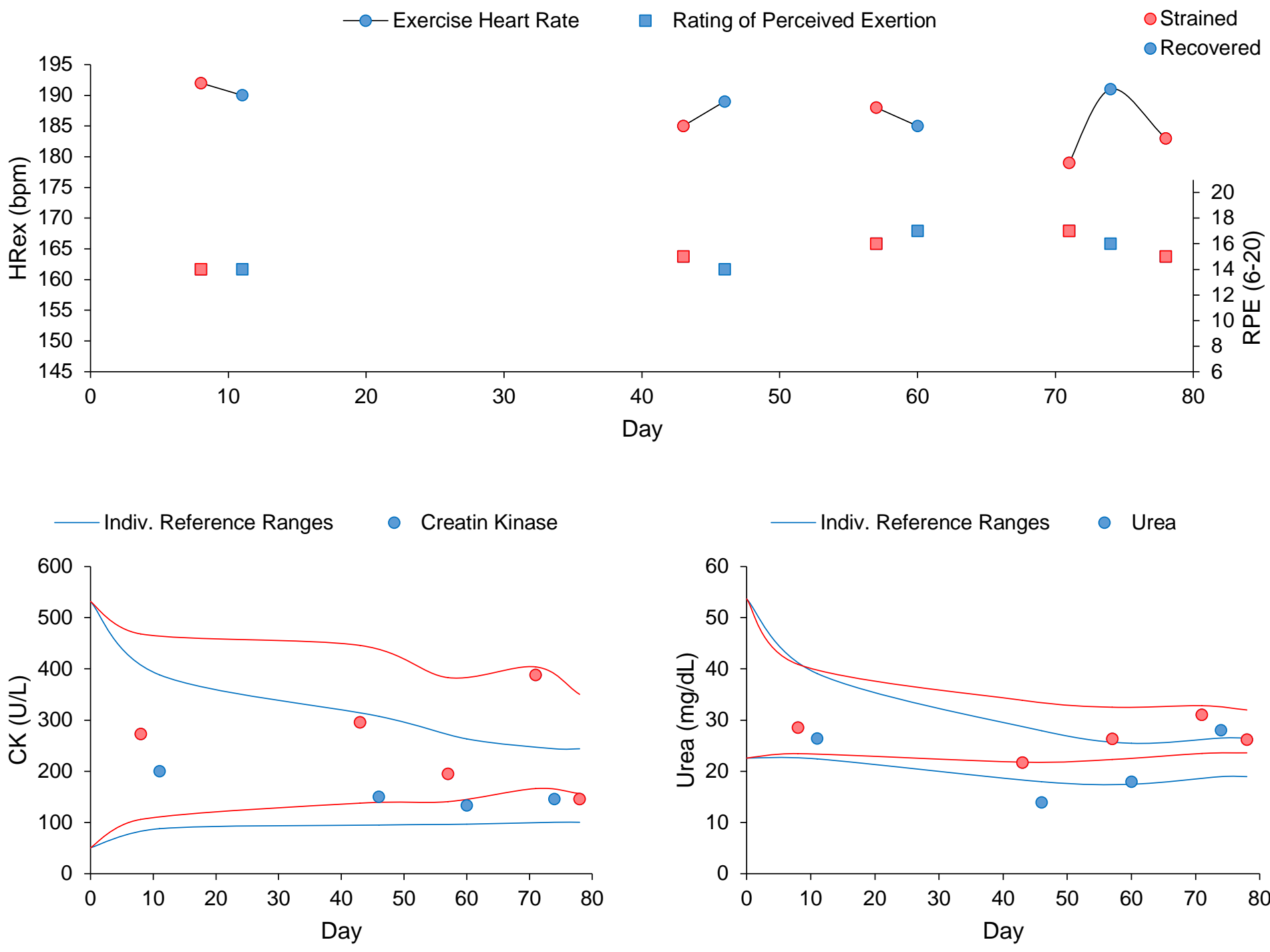

-.-- -.- Physical Perfom. Capability —-Overall Recovery
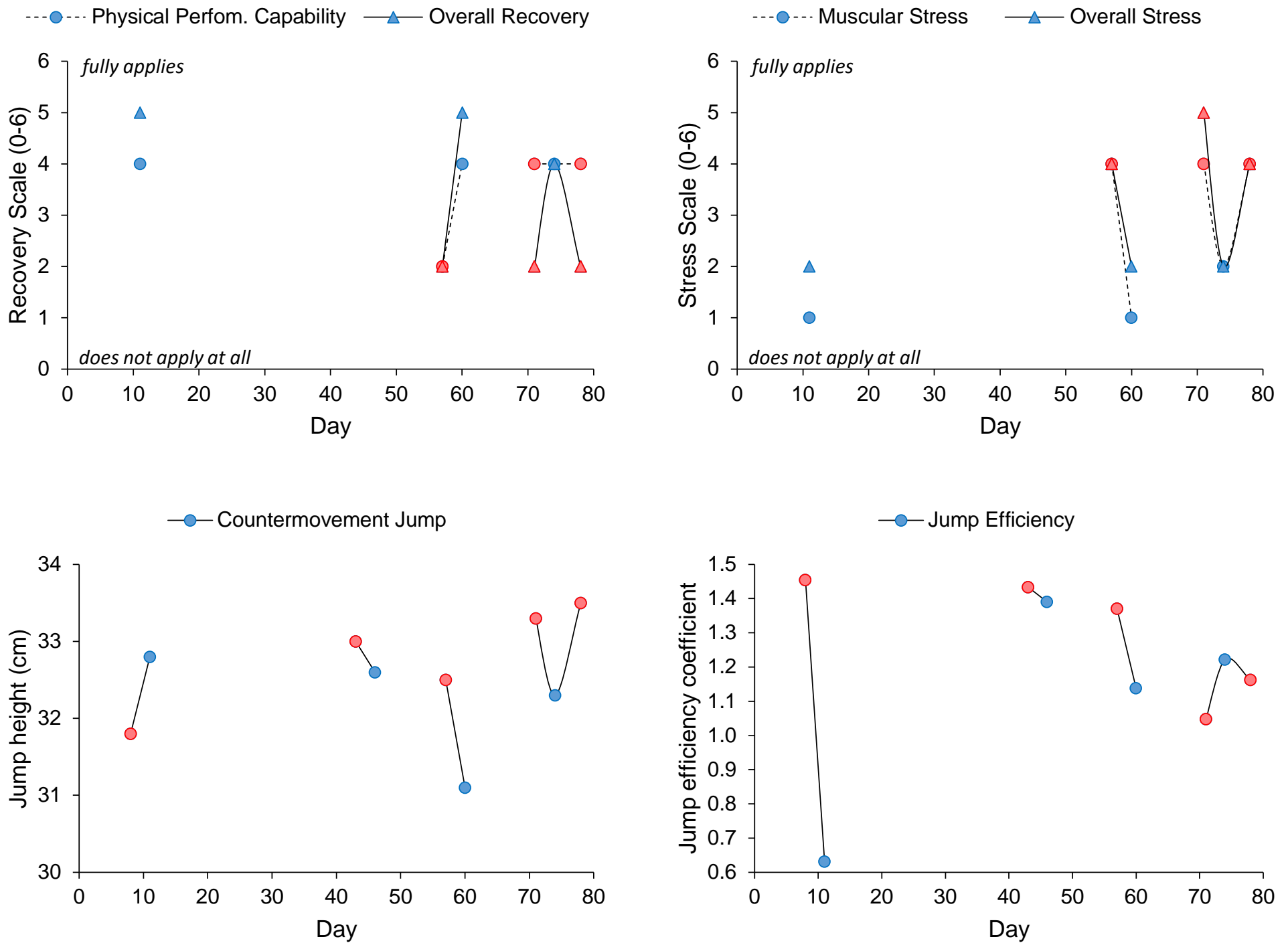

Individual data during the observational period. Blue dots: 'recovered' state (Mondays), red dots: 'strained' state (Fridays). Lines between data points are interrupted if measurements are not available or missing. HRex: exercise heart rate, RPE: rating of perceived exertion, CK: creatine kinase. Plots for CK and Urea also display individualized reference ranges for 'strained' (red lines) and 'recovered' data (blue lines) (95\% confidence intervals), see Hecksteden et al. (2017) for reference and spreadsheets: https://doi.org/10.1123/ijspp.2016-0120 


\section{Player G}
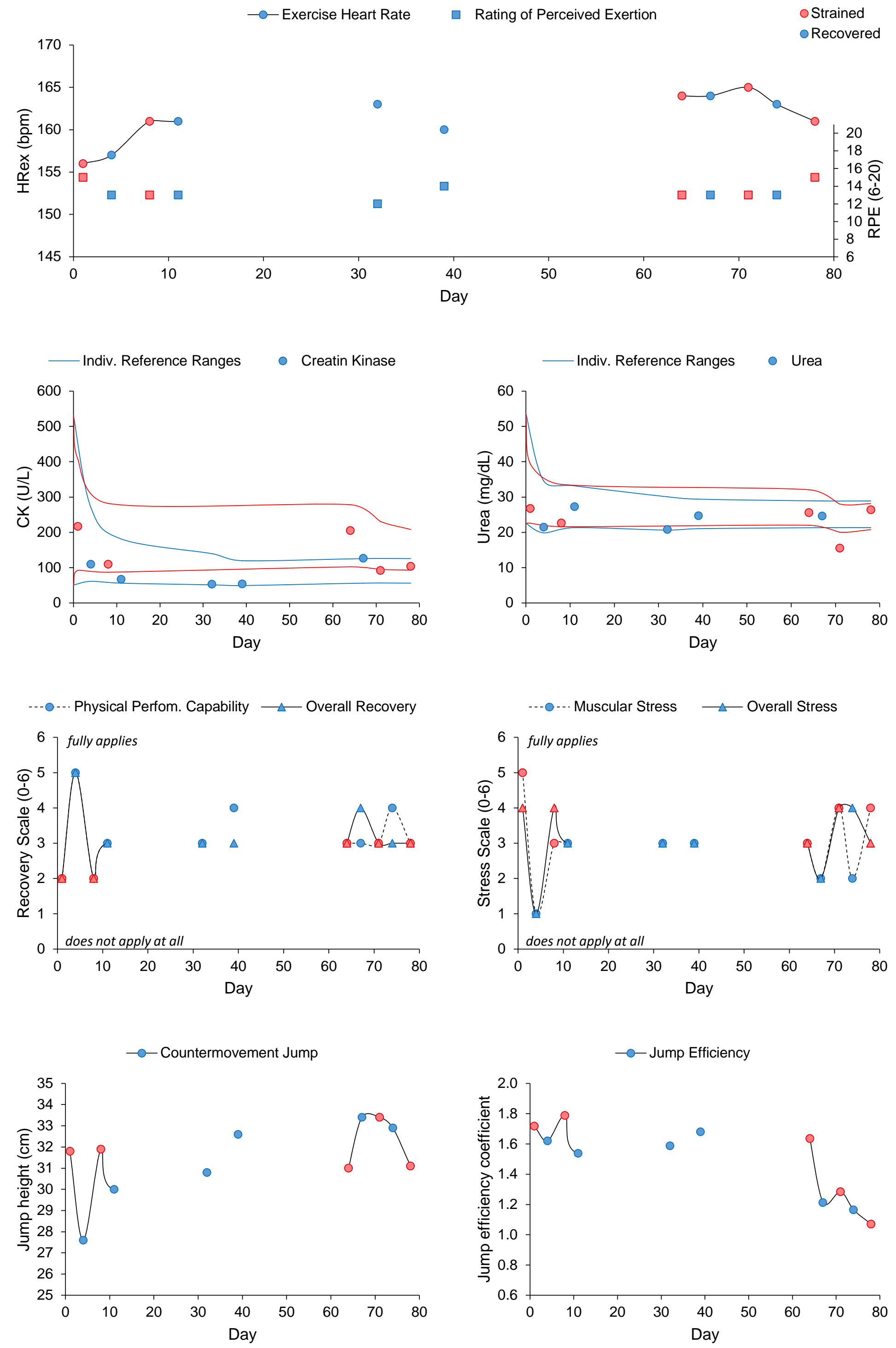

Individual data during the observational period. Blue dots: 'recovered' state (Mondays), red dots: 'strained' state (Fridays). Lines between data points are interrupted if measurements are not available or missing. HRex: exercise heart rate, RPE: rating of perceived exertion, CK: creatine kinase. Plots for CK and Urea also display individualized reference ranges for 'strained' (red lines) and 'recovered' data (blue lines) (95\% confidence intervals), see Hecksteden et al. (2017) for reference and spreadsheets: https://doi.org/10.1123/ijspp.2016-0120 


\section{Player H}
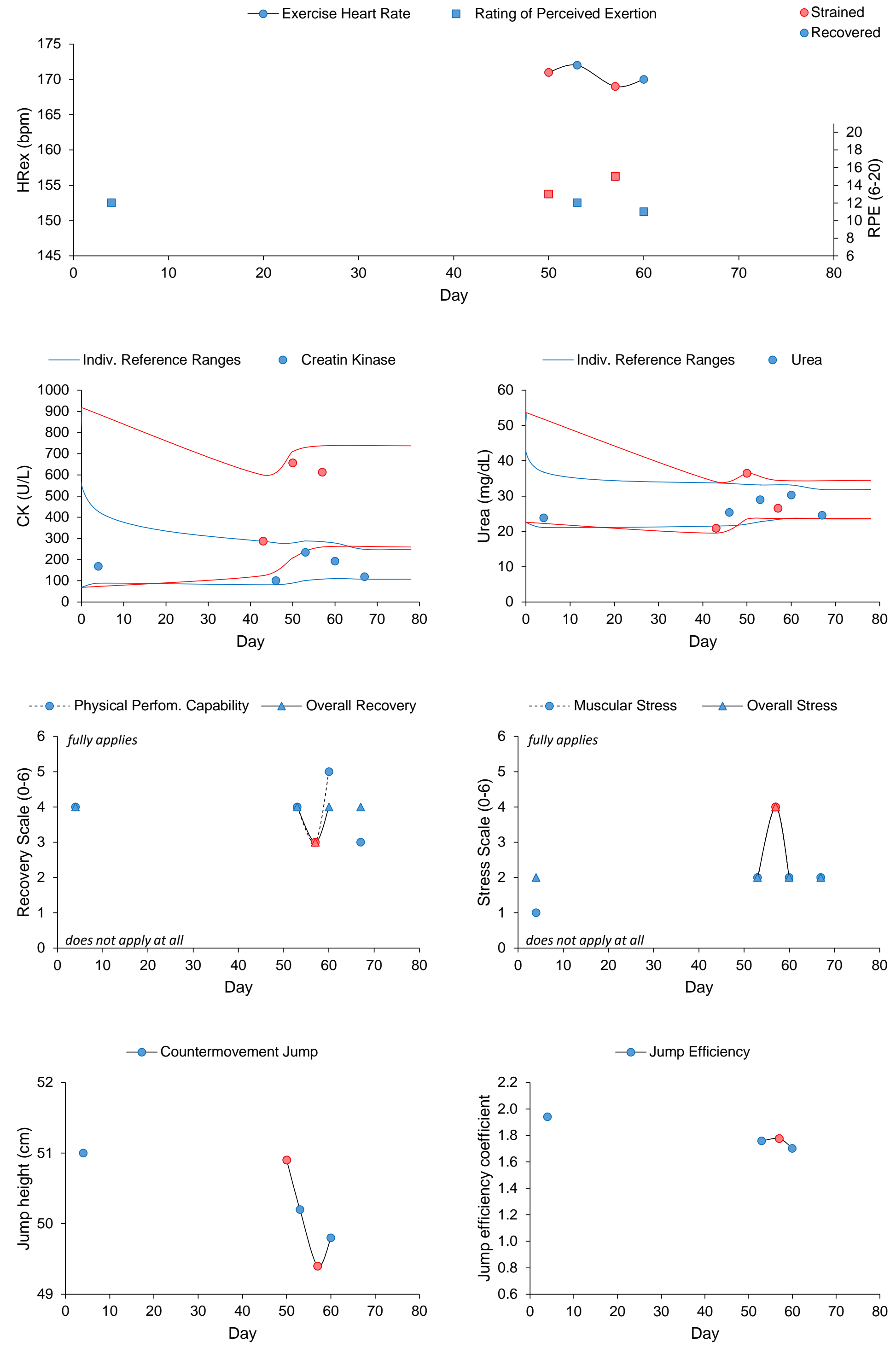

Individual data during the observational period. Blue dots: 'recovered' state (Mondays), red dots: 'strained' state (Fridays). Lines between data points are interrupted if measurements are not available or missing. HRex: exercise heart rate, RPE: rating of perceived exertion, CK: creatine kinase. Plots for CK and Urea also display individualized reference ranges for 'strained' (red lines) and 'recovered' data (blue lines) (95\% confidence intervals), see Hecksteden et al. (2017) for reference and spreadsheets: https://doi.org/10.1123/ijspp.2016-0120 


\section{Player I}
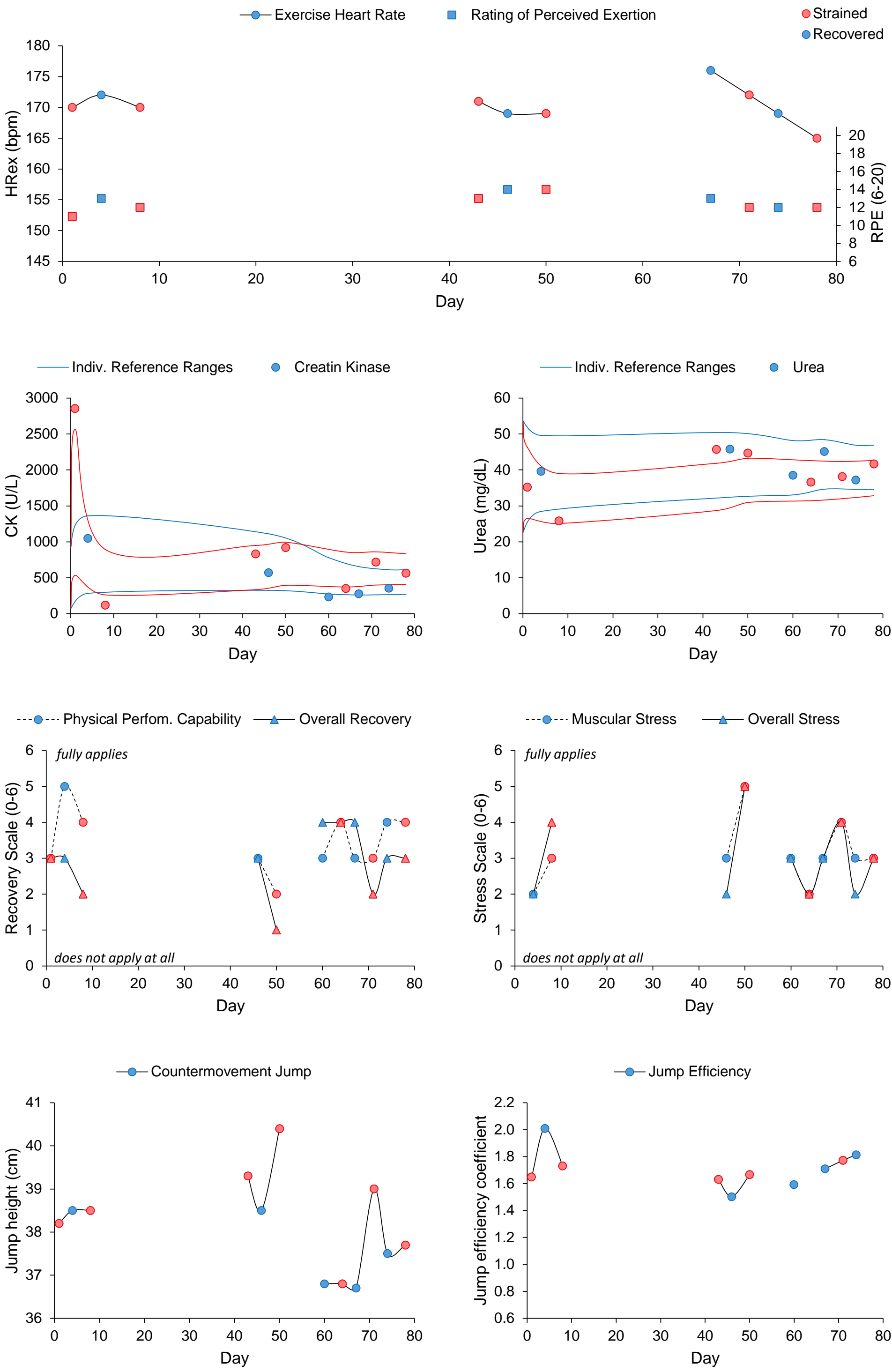

Individual data during the observational period. Blue dots: 'recovered' state (Mondays), red dots: 'strained' state (Fridays). Lines between data points are interrupted if measurements are not available or missing. HRex: exercise heart rate, RPE: rating of perceived exertion, CK: creatine kinase. Plots for CK and Urea also display individualized reference ranges for 'strained' (red lines) and 'recovered' data (blue lines) (95\% confidence intervals), see Hecksteden et al. (2017) for reference and spreadsheets: https://doi.org/10.1123/ijspp.2016-0120 


\section{Player J}
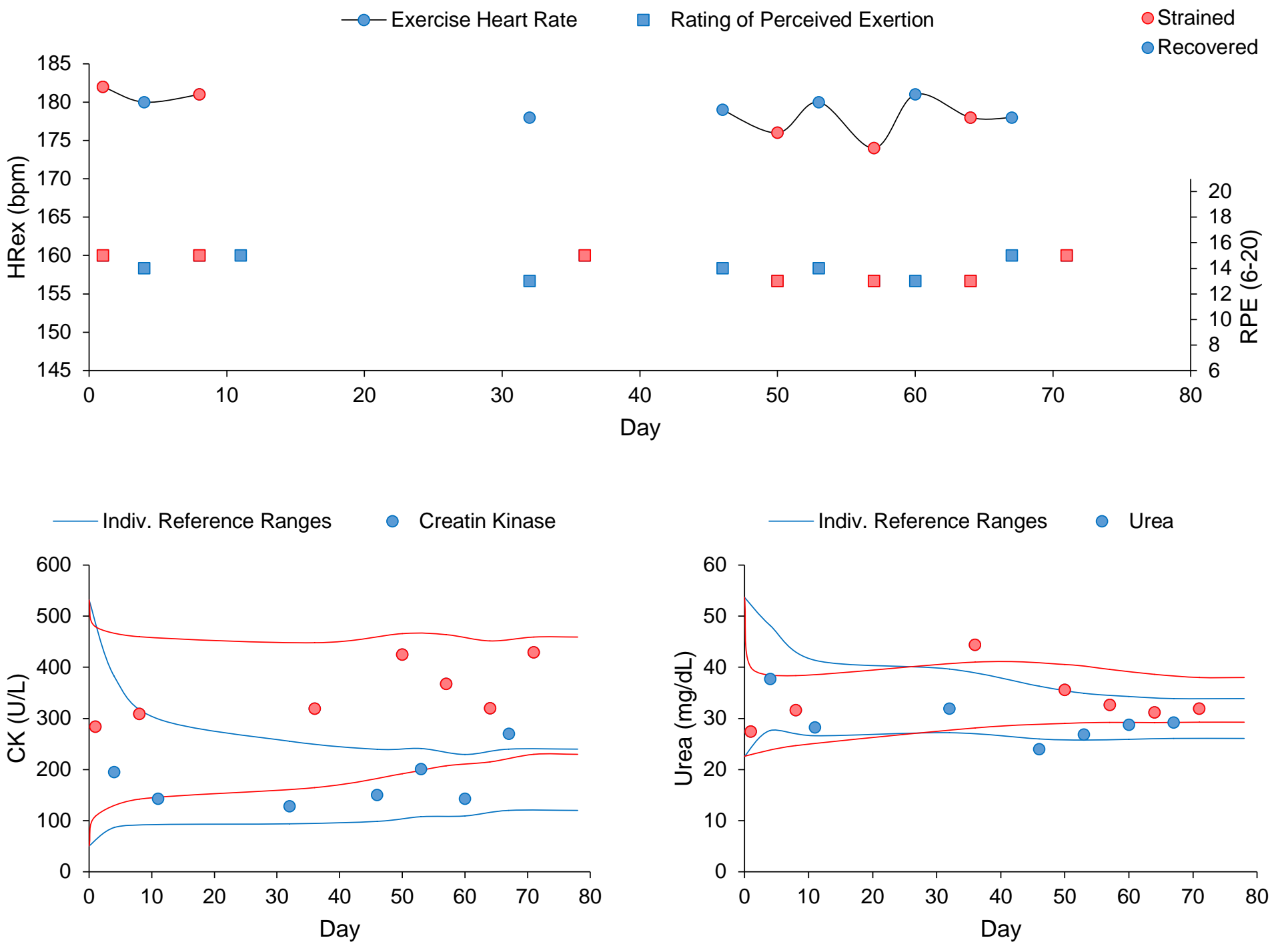

---o-.- Physical Perfom. Capability —— Overall Recovery
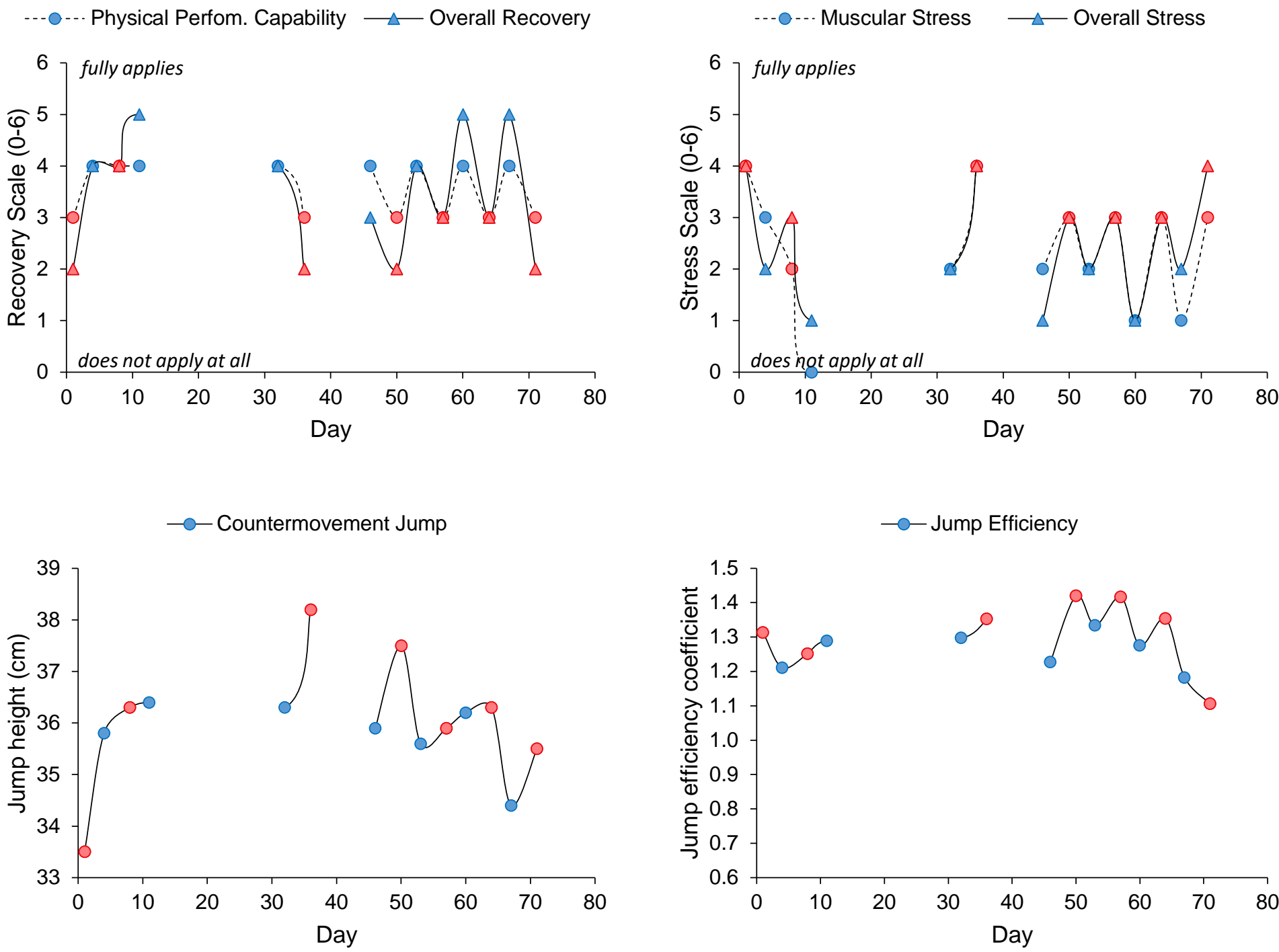

Individual data during the observational period. Blue dots: 'recovered' state (Mondays), red dots: 'strained' state (Fridays). Lines between data points are interrupted if measurements are not available or missing. HRex: exercise heart rate, RPE: rating of perceived exertion, CK: creatine kinase. Plots for CK and Urea also display individualized reference ranges for 'strained' (red lines) and 'recovered' data (blue lines) (95\% confidence intervals), see Hecksteden et al. (2017) for reference and spreadsheets: https://doi.org/10.1123/ijspp.2016-0120 